\title{
EFEITO DO FRIO NA BROTAÇÃO DE GEMAS DE PEREIRA (Pyrus communis L.) cv. Carrick, EM PELOTAS, RS ${ }^{1}$
}

\author{
FLÁVIO GILBERTO HERTER ${ }^{2}$, LILIA BENDER MACHADO ${ }^{3}$, \\ MARISA DE FREITAS OLIVEIRA ${ }^{4}$, JOÃO BATISTA DA SILVA ${ }^{5}$
}

\begin{abstract}
RESUMO - Objetivou-se, no presente trabalho, identificar a profundidade de dormência e a velocidade de brotação em gemas de pereira, submetidas a diferentes períodos de frio à temperatura de $4^{\circ} \mathrm{C} \pm 1$. O experimento foi conduzido na Embrapa-Clima Temperado, em Pelotas, em 1999. Em $1^{\circ}$ de junho, foram coletados 50 ramos, na cultivar Carrick, com aproximadamente $30 \mathrm{~cm}$ de comprimento. Após, foram divididos em 5 lotes de 10 ramos, sendo 4 mantidos a $4^{\circ} \mathrm{C} \pm 1$, e um em condições ambiente, constituindo, assim, 5 tratamentos: 0 (Testemunha); 272; 544; 816 e 1088 horas de frio (HF). No final de cada tratamento, os ramos foram divididos em pequenas estacas, contendo apenas uma única gema, sendo, após, armazenados em câmara climática a $25^{\circ} \mathrm{C} \pm 1$. Avaliou-se a brotação, considerando-se o estádio de ponta verde. A partir destes dados, calculou-se o tempo médio de brotação (TMB), bem como a percentagem de gemas brotadas, em cada um dos tratamentos. Utilizou-se o índice de velocidade de brotação (IVB), para determinar a eficiência da temperatura na brotação das gemas. A profundidade de dormência, das gemas terminais, diminuiu à medida que se aumentou $\mathrm{O}$ período de frio. As gemas axilares não foram influenciadas pelo tempo de exposição ao frio. Com base nos dados do IVB e dos coeficientes angulares, as gemas terminais da cv. Carrick necessitam de 800 horas de frio para completar a brotação, nas condições que foram conduzidos os experimentos.
\end{abstract}

Termos para indexação: Temperatura, dormência, Pyrus communis, tempo médio de brotação, método biológico.

\section{EFFECT OF CHILLING ON THE BUD BREAKING OF PEAR CV. CARRICK, IN PELOTAS, RS.}

\begin{abstract}
The objective for this work was to identify the dormancy depth and the bud-sprouting rate of pear trees kept at chilling conditions $\left(4^{\circ} \mathrm{C} \pm 1\right)$ for different periods. The experiment was carried out using buds of twigs of the previous growth season from a pear orchard of the Embrapa Clima Temperado Research Center. The twigs were collected on June 1, 1999. The treatments were five period of chilling: 0 (control); 272; 544; 816; or 1088 hours at $4^{\circ} \mathrm{C} \pm 1$. At the end of each treatment, the twigs were cut into short cuttings containing one lateral or terminal bud each and placed in controlled conditions at $25^{\circ} \mathrm{C} \pm 1$. The variables evaluated were percentage of bud break and period of time to reach that phase. The mean of time of bud break (MTB) for the terminal buds decreased significantly on the cuttings that received 816 or 1088 chilling hours which were equivalent on MTB. Similarly the bud break rate (time to reach $100 \%$ bud break) was significantly shorter on the buds that received 816 or 1088 chilling hours. Therefore the terminal buds of pear cv. Carrick need 800 hours for a satisfactory bud break.
\end{abstract}

Index Terms: Temperature, dormancy, Pyrus communis, mean time to bud break (MTB), biological method.

\section{INTRODUÇÃO}

No Sul do Brasil, existem regiões com condições edafoclimáticas propícias para o cultivo da pereira. Testes realizados com coleções de cultivares mostraram que a cv. Carrick apresenta boa adaptação, constituindo-se numa alternativa promissora para os produtores. Entretanto, fazem-se necessários estudos mais detalhados sobre seu período de dormência.

A superação da dormência, quando não se dá de forma adequada, pode causar alterações na brotação, através da morte de gemas com conseqüente queda na produção (Barnola et al., 1976).

As gemas das frutíferas temperadas durante a fase de repouso se caracterizam por apresentar um estado de maior ou menor profundidade de dormência, dependente da interação do genótipo com o ambiente (Barnola et al., 1976).

Embora exista o método bioquímico (teste de nucleotídeos) para caracterizar o nível de dormência, o teste biológico é o único que quantifica a profundidade de dormência. Este método vem sendo utilizado com bastante freqüência em frutíferas temperadas, tais como pessegueiros (Bonhomme et al., 1999; Nishimoto \& Fujisaki, 1995), macieira (Herter et al., 1992), pereiras (Nishimoto \& Fujisaki, 1995) e videira (Nishimoto \& Fujisaki, 1995). Este é baseado no princípio da inibição correlativa, onde uma gema tem ação sobre a outra. Entretanto, quando se utilizam estacas, formadas a partir de hastes, contendo

\footnotetext{
1 Trabalho $\mathrm{n}^{\mathbf{0}}$ 008/2001. Recebido: 08/01/2001. Aceito para publicação: 17/07/2001.Apoio financeiro EMBRAPA, CNPq e JICA (Projeto de Horticultura para Pequenos produtores no Sul do Brasil).

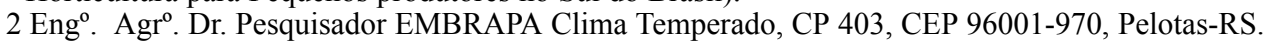

3 Eng. $^{\circ}$ Agr ${ }^{\circ}$. Mestrando em Agronomia, FAEM, UFPel, Pelotas-RS.

4 Eng $^{\circ}$. Agr ${ }^{\circ}$. Msc., Doutorando em Agronomia, FAEM, UFPel, Pelotas-RS.

5 Eng ${ }^{\circ}$. Agr ${ }^{\circ}$., Dr., livre docente, prof. Titular IMF-UFPel, Pelotas-RS.
} 
uma única gema, este efeito é eliminado, e a gema poderá desenvolver todo seu potencial (Champagnat et al., 1983).

O trabalho objetivou determinar a profundidade da dormência das gemas terminais e axilares de pereira cv. Carrick, submetidas a diferentes quantidades de frio, bem como a velocidade de brotação a $25^{\circ} \mathrm{C} \pm 1$.

\section{MATERIAL E MÉTODOS}

O material vegetal utilizado no experimento, a cultivar Carrick, é originário dos Estados Unidos da América, sendo um híbrido proveniente do cruzamento das cvs. Garber x Seckel. Apresenta frutos de tamanho médio com formato periforme, coloração marrom-esverdeada, com manchas avermelhadas na epiderme e polpa branca, sabor e qualidade regulares (Ribeiro et al., 1991).

Foram coletados, no dia 10-6-1999, 50 ramos de plantas pertencentes a uma coleção instalada na Estação Experimental da Cascata, Embrapa Clima Temperado, em Pelotas, no Rio Grande do Sul. Após a coleta, os ramos foram armazenados em câmara frigorífica a uma temperatura de $4 \pm 1^{\circ} \mathrm{C}$, por períodos determinados de acordo com os seguintes tratamentos: ausência de frio, 272; 544; 816 e 1088 horas de frio (HF).

No final de cada tratamento, 10 ramos foram seccionados em estacas com aproximadamente $8 \mathrm{~cm}$, permanecendo somente a gema distal superior de cada estaca. Para diminuir o efeito da desidratação, parafinou-se a porção superior de cada estaca (exceto a porção que continha a gema apical). A seguir, as estacas foram acondicionada em bandejas mantida em fitotron, à temperatura de $25^{\circ} \mathrm{C} \pm 1$ e 14 horas de luz, para forçar a brotação. A avaliação da brotação foi realizada, semanalmente, anotandose a data de brotação, no estádio de ponta verde.

\section{Tempo Médio de Brotação (TMB)}

O tempo médio de brotação é o número de dias passado entre a coleta do material vegetal, em cada época, e a detecção do estádio de ponta verde (PV). O TMB correspondente ao lote de estacas, para cada um dos tratamentos, foi estimado pela expressão:

$\mathrm{TMB}=\sum \mathrm{n}_{\mathrm{i}} \mathrm{t}_{\mathrm{i}} / \mathrm{n}$, onde $\mathrm{n}_{\mathrm{i}}=$ número de gemas brotadas no tempo $\mathrm{t}_{\mathrm{i}}$ $\mathrm{t}_{\mathrm{i}=}$ tempo decorrido a partir do condicionamento a $25^{\circ} \mathrm{C} \pm 1$, até a brotação; $\mathrm{n}=$ número total de gemas brotadas.

\section{Índice de Velocidade de Brotação (IVB)}

O IVB, índice de velocidade de brotação, é um índice proposto para quantificar a capacidade de brotação das gemas de plantas frutíferas, medida que é análoga ao índice de velocidade de germinação (IVG) proposto por Amaral (1979) para calcular o poder germinativo das sementes.

O índice de velocidade de brotação foi calculado através da seguinte fórmula proposta por Amaral (1979):

$$
\mathbf{I V B}=\mathbf{n} / \sum \mathbf{n}_{\mathbf{i}}\left(\mathbf{1} / \mathbf{d}_{\mathbf{i}}\right)
$$

Onde: $\mathrm{n}=$ número de gemas brotadas; $\mathrm{n}_{\mathrm{i}}=$ número de gemas brotadas na data $\mathrm{i} ; \mathrm{d}_{\mathrm{i}}=$ dias até a brotação.

\section{RESULTADOS E DISCUSSÃO}

O frio causou modificações tanto na profundidade de dormência, como na velocidade de brotação (Figuras 1 e 3, respectivamente). Observa-se, na Figura 1, que a profundidade de dormência das gemas terminais diminuiu à medida que se aumentou o acúmulo de horas de frio. Neste caso, quando as gemas terminais foram expostas a 816 e 1088 horas de frio, a superação da dormência deu-se em menor tempo, comparado aos demais tratamentos. Pode ser ainda observado nesta mesma figura, que as gemas expostas ao período de 816 horas de frio apresentaram TMB mais uniforme, representado pelo menor desvio-padrão.

O efeito do frio é conhecido na eliminação da dormência; entretanto, o seu real mecanismo, considerando-se os aspectos fisiológicos, resta, ainda, ser aprofundado. Sabe-se que a temperatura intervém ao nível de membrana, direta ou inderetamente, estimulando as modificações da composição

TABELA 1 - Número de gemas terminais brotadas de pereira cv. Carrick. Embrapa Clima Temperado, Pelotas-RS, 2000.

\begin{tabular}{|c|c|c|c|c|c|c|c|c|c|c|c|c|c|}
\hline \multirow{2}{*}{\begin{tabular}{|l} 
H oras $\mathrm{de}$ \\
F rio \\
\end{tabular}} & \multicolumn{13}{|c|}{ D ias a té a brotação } \\
\hline & 4 & 7 & 10 & 13 & 16 & 19 & 22 & 25 & 28 & 31 & 34 & 37 & 40 \\
\hline 0 & & & & & & 3 & 3 & 3 & 3 & 7 & 7 & 10 & \\
\hline 544 & & & & 3 & 8 & 8 & 8 & 9 & 9 & 10 & & & \\
\hline 816 & & 2 & 6 & 9 & 10 & & & & & & & & \\
\hline 1088 & 5 & 5 & 6 & 10 & & & & & & & & & \\
\hline
\end{tabular}

TABELA 2 - Distribuição do número de gemas terminais brotadas, em dias, até a data de brotação, em pereira, cv. Carrick. Embrapa Clima Temperado, Pelotas-RS, 2000.

\begin{tabular}{|c|c|c|c|c|c|c|c|c|c|c|c|c|c|c|}
\hline \multirow{2}{*}{$\begin{array}{l}\text { H oras } \\
\text { de Frio }\end{array}$} & \multicolumn{14}{|c|}{ Inverso do número de dias até a brotação $\left(1 / d_{i}\right)$} \\
\hline & $1 / 4$ & $1 / 7$ & $1 / 10$ & $1 / 13$ & $1 / 16$ & $1 / 19$ & $1 / 22$ & $1 / 25$ & $1 / 28$ & $1 / 31$ & $1 / 34$ & $1 / 37$ & $1 / 40$ & IV B \\
\hline 0 & & & & & & 3 & & & & 4 & 3 & & & 27,2 \\
\hline 272 & & & & & 2 & 2 & & 2 & & & & & & 24,4 \\
\hline 544 & & & & 3 & 5 & & & 1 & & 1 & & & & 16,2 \\
\hline 816 & & 2 & 4 & 3 & 1 & & & & & & & & & 10,2 \\
\hline 1088 & 5 & & 1 & 4 & & & & & & & & & & 6,0 \\
\hline
\end{tabular}




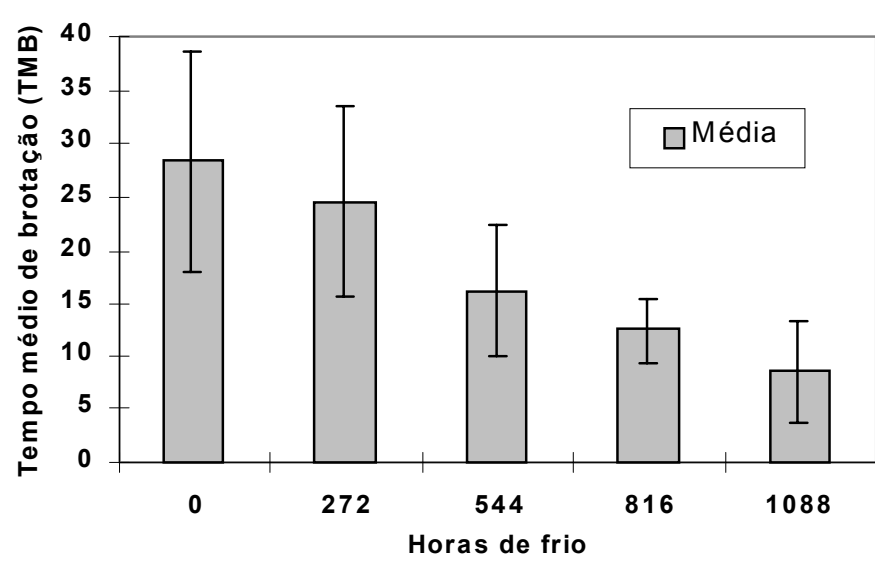

FIGURA 1 - Tempo médio de brotação em gemas terminais de pereira cv. Carrick. Embrapa Clima Temperado, Pelotas-RS, 2000. (As barras, em cada coluna, expressam o desvio-padrão).

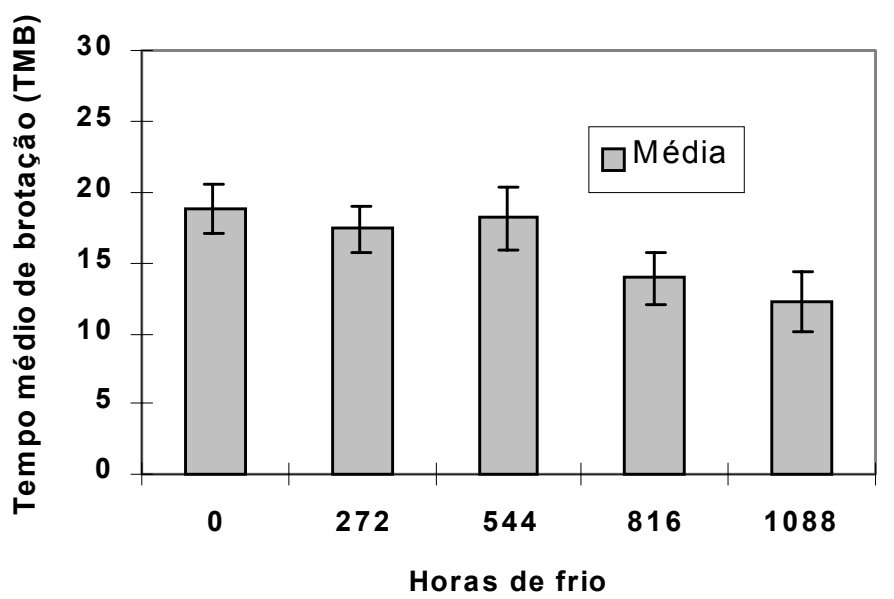

FIGURA 2 - Tempo médio de brotação em gemas axilares de pereira cv. Carrick. Embrapa Clima Temperado, Pelotas-RS, 2000. (As barras, em cada coluna, expressam o desvio-padrão).

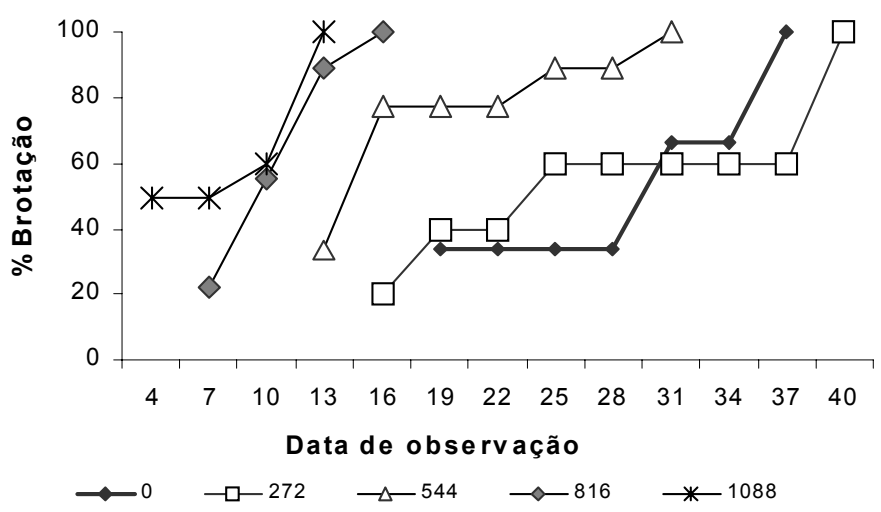

FIGURA 3 - Percentagem de brotação em gemas terminais de pereira cv. Carrick. Embrapa Clima Temperado, Pelotas-RS,2000. protéica e lipídica (Mazliak, 1992; Portrat et al., 1995); intervém, ainda, na velocidade das reações enzimáticas, na respiração e nos fenômenos de transporte (Lance e Moreau, 1992).

Para o caso das gemas laterais, o TMB não foi influenciado pelo tempo de exposição ao frio (Figura 2).

No caso de pomáceas, principalmente macieira e pereira, as gemas terminais exercem um importante papel no controle da dormência sobre as gemas laterais. Neste caso, apresentam maior profundidade de dormência (Herter, 1992), necessitando, portanto, de maior período de exposição ao frio.

Em relação à velocidade de brotação (Figura3), esta foi diretamente proporcional às quantidades de frio. Entretanto, os tratamentos 816 e 1088 horas de frio, embora representem apenas 272 horas de frio, de diferença, podem ser considerados de igual efeito, pois, em cerca de 15 dias, as gemas atingiram o índice $100 \%$ de brotação. Para o caso das gemas submetidas a $544 \mathrm{HF}$, ocorreu um aumento rápido, passando de $30 \%$ para $80 \%$ de brotação, num período de 2 dias, após 14 dias de exposição, chegando a $100 \%$ somente aos 30 dias. Já na testemunha e 272 HF, o índice de $100 \%$ de gemas brotadas ocorreu aos 38 e 29 dias após a retirada do frio, respectivamente.

No tabela 1, são apresentados os dados do número de gemas terminais brotadas a partir do início da brotação, em função do tratamento de frio submetido. Na tabela 2, é apresentada a distribuição do número de gemas terminais brotadas, a partir do qual se calculou o IVB.

De acordo com os resultados do quadro 2, pode-se ver que o tratamento de 1088 horas de frio indicou um IVB de cerca de 6,0 dias e o tratamento de 816 horas de frio, de 10,2 dias de IVB. Os dois tratamentos diferiram por cerca de 4 dias. Os demais tratamentos apresentaram os IVBs respectivos, com, no mínimo o dobro daquele do tratamento de $1088 \mathrm{HF}$.

A partir dos dados do quadro 1 e Figura 3, avaliando a regressão linear do número de gemas terminais brotadas $(\mathrm{Y}) \mathrm{em}$ relação aos das de brotação $(\mathrm{X})$, podem-se estimar as seguintes equações, para cada tratamento:
1) $0 \mathrm{HF}$
$\mathrm{Y}_{\mathrm{i}}=0,43+1,2 \mathrm{X}_{\mathrm{i}}$
$\mathrm{r} 2=0,80$
2) $272 \mathrm{HF}$
$\mathrm{Y}_{\mathrm{i}}=2,1+0,7 \mathrm{X}_{\mathrm{i}}$
$\mathrm{r} 2=0,77$
3) $544 \mathrm{HF}$
$\mathrm{Y}_{\mathrm{i}}=4,4+0,86 \mathrm{X}_{\mathrm{i}}$
$\mathrm{r} 2=0,67$
4) $816 \mathrm{HF}$
$\mathrm{Y}_{\mathrm{i}}=2,7 \mathrm{X}_{\mathrm{i}}$
$\mathrm{r} 2=0,94$
5) $1088 \mathrm{HF}$
$\mathrm{Y}_{\mathrm{i}}=2,5+1,6 \mathrm{X}_{\mathrm{i}}$
$\mathrm{r} 2=0,75$
$\mathrm{X}_{\mathrm{i}}=(1,2,3 \ldots$.conforme
o caso)

Poder-se-iam agrupar os tratamentos pela magnitude dos coeficientes angulares (que indicam a intensidade da resposta de $\mathrm{Y}$ a cada variação de X), em 3 grupos: 2 e 3, no grupo de menor intensidade de resposta; 1 e 5 , no grupo de intensidade intermediária; 4, no de maior intensidade. $O$ teste de significância (teste $\mathrm{t}$ ) entre as inclinações das retas indicou diferença significativa do tratamento de $816 \mathrm{HF}$ em relação ao tratamento de $544 \mathrm{HF}(\alpha=0,05)$, em relação ao tratamento de $272 \mathrm{HF}$ $(\alpha=0,01)$ e em relação ao tratamento de $0 \mathrm{HF}(\alpha=0,06)$, segundo Gomez e Gomez (1984).

A brotação das gemas terminais foi mais rápida quando se utilizaram 816 horas de frio. Resultados de pesquisa têm mostrado que o frio provoca a homogeneização da dormência, pois as gemas terminais, durante a fase de repouso, apresentam diferentes níveis de profundidade (Mauget, 1987). Deve-se salientar que a avaliação da velocidade da brotação foi determinada após as gemas terem sido submetidas ao calor 
$\left(25^{\circ} \mathrm{C}\right)$. Portanto, estes resultados indicam que o frio aumentou a velocidade de brotação.

A partir destes resultados, é possível estimar que as gemas terminais da cv. Carrick necessitam de uma exposição de cerca de 800 horas de frio para superar a dormência.

\section{CONCLUSÕES}

1 - O estado inercial das gemas terminais diminuiu com o aumento do número de horas de frio a que foram submetida.

2 - O índice de velocidade de brotação do tratamento de 1088 horas de frio, o de menor índice, não diferiu do tratamento de 816 horas de frio, mas foi inferior aos demais.

3 - O índice de velocidade de brotação pode ser utilizado para estimar a necessidade de frio das cultivares.

4 - A intensidade de brotação a 816 horas de frio é superior às demais.

5 - As gemas da cv. Carrick necessitam de uma exposição de 800 horas de frio para superar a dormência.

\section{REFERÊNCIAS BIBLIOGRÁFICAS}

AMARAL, E. Alguns problemas de Estatística aplicada em análise de sementes. Tecnologia de Sementes, Pelotas, UFPe1, v.2, n.1, p.12-18, 1979.

BARNOLA, P.; CHAMPAGNAT, P.; LAVARENNE, S. Taille en vert des rameaux et dormance des bourgeons chez le noisetier. Cahiers Recherche Acad. Agriculture France., 62, 1163-1171, 1976.

BONHOMME, M.; RAGEAU, R.; RICHARD, J. P.; EREZ, A.; GENDRAUD, M. Influence of three contrasted climatic conditions on endodormant vegetative and floral peach buds: analyses of their intrinsic growth capacity and their potential sink strength compared with adjacent tissues. Scientia Horticulturae, Amsterdan, n.80, 1999.

CHAMPAGNAT, P. Bud dormancy, correlation between organs, and morphogenesis in woody plants. Fiziologiya Rastenii, Moxow, v.30, n.30, 1983.
GOMEZ, K. A.; GOMEZ, A. A. Statistical procedure for agricultural research. 2., John Wiley \& Sons, 1984. 680 p.

HERTER, F. G. Dormance des bourgeons et phenologie des quelques cultivars de pommier: effet de la température en interaction avec le génotype. 1992, 82 f. Tese (Doutorado) Université Blaise Pascal, Clermont Fd. II. 1992.

HERTER, F.G.; RAGEAU, R.; BONHOMME, M.; MAUGET, J.C. Determinação do término da dormência e floração para alguns cultivares de macieira: comparação entre métodos biológicos e empíricos. Revista Brasileira de Fruticultura, v.14, n..1, 1992.

LANCE, C.; MOREAU, F. Les effets métaboliques du froid. In: HERMANN, C. D. (Ed.) Les vegetaux et le froid. Paris, 1992, p. 27-49.

MAUGET, J.C. Dormance des bourgeons chez les arbres fruitiers de climat tempéré. In: LE GUYADER H. (Ed.), Le développement des végétaux. Aspects théoriques et synthétiques. Paris: Masson, p. 133-150.

MAZLIAK, P. Les effets du froid sur les biomembranes. In: Les végétaux et le froid. Côme D. Hermann (Ed), Paris (FRA), 326. 1992.

NISHIMOTO, N.; FUJISAKI, M. Chilling requirement of buds of some deciduous fruits grown in southern Japan and the means to break dormancy. Acta Horticulturae, Wageningen, n.395, 1995.

PORTRAT K., MATHIEU C., MOTTA C., PETEL G. Changes in plasma membrane properties of peach tree buds and stands during dormancy. Journal Plant Physiology, Stuttgart, v. 147, p. 346-350. 1995

RIBEIRO, P. de A.; BRIGHENTI, E.; BERNARDI, J. Comportamento de algumas cultivares de pereira Pyrus communis L. e suas características nas condições do Planalto Catarinense. Florianópolis: EMPASC, 1991. 53. (Boletim Técnico, 56). 